\title{
Pharmacokinetic Concentration Reason Not Done
}

National Cancer Institute

\section{Source}

National Cancer Institute. Pharmacokinetic Concentration Reason Not Done. NCI

Thesaurus. Code C87963.

The explanation given as to why pharmacokinetic concentration was not assessed. 